\title{
Stabilization of the Dispersed System of Halloysite Nanotubes for Silicate Constructional Material
}

\author{
Natalia Lukuttsova ${ }^{1,}{ }^{*}$, Sergey Golovin ${ }^{1}$, Natalia Zolotukhina ${ }^{2}$, and Olga Sycheva ${ }^{1}$ \\ ${ }^{1}$ Bryansk State Engineering and Technology University, Bryansk, Russia \\ ${ }^{2}$ Bender Polytechnic University, Bender, Moldova
}

\begin{abstract}
The stabilization of the dispersed system of halloysite nanotubes $(\mathrm{HN})$ obtained by ultrasonic treatment (UST) in the aqueous medium of the stabilizers of sodium polynaphthalene methylene sulfonate stabilizers (S-3) and a synthetic compound based on polycarboxylate ether (MG) is considered. The morphology of halloysite is studied. Various mechanisms of aggregate stability connected with the spatial obstacles to aggregation due to the action of electrostatic, adsorption-solvate, and structural-mechanical stabilization factors are established. Three variants of introducing S-3 and MG stabilizers into the dispersed system of halloysite nanotubes are considered. It has been found that the most preferred method is the one with the stabilizer added in two steps. In this variant halloysite nanotubes are of minimum size and with maximum specific surface area. The maximum $\zeta$-potential values of $52.9 \mathrm{mV}$ and $43.8 \mathrm{mV}$ are obtained for the dispersed system stabilized with S-3.
\end{abstract}

For the dispersed system stabilized with $\mathrm{MG}$, the $\zeta$-potential values do not exceed $20.9 \mathrm{mV}$, while the particle sizes decrease and the specific surface area grow. This confirms the formation of spatial obstacles to aggregation due to the action of adsorption-solvate and structural-mechanical factors. The electrostatic factor of aggregate stability for these dispersed systems appears not to be crucial.

Owing to the stricter requirements for the operational and environmental characteristics of silicate composite materials, it is necessary to develop fundamentally new approaches for their production. Modern materials based on synthetic fullerenes, carbon nanotubes and others are able to meet the requirements of different industries from a technical point of view, but their high cost, minor production volumes, and in some cases high toxicity, do not allow them to be considered as a serious alternative to traditional materials and components in the years ahead. Nanotubes from the natural mineral halloysite are a promising component for most silicate systems. The advantages of raw materials for halloysite nanotubes production include low cost, sufficient volumes of output and good compatibility with the basic components of silicate materials.

\footnotetext{
*Corresponding author: natluk58@mail.ru
} 
Nevertheless, the uneven distribution of nano- and microdisperse additives in the silicate structural composites and unstable aggregate and sedimentation stability hamper their wide-ranging use.

The aim of the work is to study the stabilization of the dispersed system of halloysite nanotubes for silicate structural materials.

In the research the halloysite nanotubes NTH (Russia, Yuzhnouralsk) are studied. It is light yellow powder with a density of $2590 \mathrm{~kg} / \mathrm{m}^{3}$, a specific surface area of $499 \mathrm{~m}^{2} / \mathrm{kg}$, a modal diameter of 50.62 microns, an average particle diameter of 32.3 microns with a span (d90-d10)/d50 $=2.47$ microns ranging from 0.01 to 2000 microns.

The chemical composition of the halloysite nanotubes was determined by X-ray fluorescence spectrometry with OPTIM-X spectrometer. It is established that a typical elementary composition of $\mathrm{HN}$ consists of silicon $(22.15 \%)$; aluminium $(25.32 \%)$; potassium $(1.69 \%)$; iron $(1.11 \%)$; calcium $(0.06 \%)$; lead $(0.19 \%)$; manganese $(0.13 \%)$; titanium $(0.05 \%)$; phosphorus $(0.03 \%)$ and others. The oxide composition is represented by the basic oxides: $\mathrm{SiO}_{2}-48.85 \%$ и $\mathrm{Al}_{2} \mathrm{O}_{3}-45.16 \%$. Besides, the composition of halloysite nanotubes includes the following oxides $(\% \mathrm{wt}): \mathrm{K}_{2} \mathrm{O}-2.11, \mathrm{Fe}_{2} \mathrm{O}_{3}-1.16, \mathrm{CaO}-0.26$, $\mathrm{PbO}-0.23, \mathrm{MnO}-0.18, \mathrm{TiO}_{2}-0.09, \mathrm{P}_{2} \mathrm{O}_{5}-0.08$ and others.

Regarding the structure and chemical composition, halloysite is referred to the group of clay minerals, the subclass of layered silicates. It is extracted from kaolinite deposits. It consists of aluminium hydrosilicate $\mathrm{Al}_{2} \mathrm{Si}_{2} \mathrm{O}_{5}(\mathrm{OH})_{4} \cdot \mathrm{nH}_{2} \mathrm{O}$. Halloysite crystallizes into monoclinic syngony. It is characterized by waxy and porcelain-like aggregates. The halloysite colour is white, light yellow, gray, bluish. Its mineralogical hardness number ranges from 1 to 2.5 [1-3].

Halloysite is distinguished from kaolinite by the higher water content (the aluminosilicate layers are separated by $\mathrm{H}_{2} \mathrm{O}$ molecules). The water layer in halloysite is loosely bound: there is about $1 \mathrm{~nm}$ between the layers. At dehydration of halloysite the distance between the layers decreases to $0.7 \mathrm{~nm}[4,5]$.

Owing to the discrepancy in the periods of the tetrahedral outer layer of $\mathrm{SiO}_{2}$ and the inner layer of $\mathrm{Al}_{2} \mathrm{O}_{3}$ with an octahedral configuration, a layered tubular structure is formed $[6,7]$.

Using the method of scanning electron microscopy (SEM) with TESCAN MIRA 3 LMU microscope (Czech Republic), it was found that halloysite nanotubes have an outer diameter of up to $300 \mathrm{~nm}$, an inner diameter ranging from 20 to $100 \mathrm{~nm}$, and a length of 50$50,000 \mathrm{~nm}$ (Fig. 1).

$\mathrm{Al}-\mathrm{OH}$ groups are located on the inner surface of the nanotubes, while $\mathrm{Si}-\mathrm{O}-\mathrm{Si}$ groups are placed on the outer surface $[8,9]$. In this case, the outer surface is negatively charged, and the inner surface has a positive charge (Fig. 2a). The multilayer nature of the halloysite nanotube in the form of a spiral ribbon is clearly shown in Figure $2 b$. The sharp edges of the nanotube chips with a bare inner surface are visible.

The dispersion of halloysite nanotubes was obtained by ultrasonic dispersion in the bath-type activator USD-13/150-TN-RELTEC at an ultrasound frequency of $44 \mathrm{kHz}$ in the aqueous medium of the stabilizer.

The stabilizers (the S-3 and MG additives), varied by mechanism of action, were studied for the aggregate stability of the dispersed system based on halloysite nanotubes.

The S-3 additive refers to sodium polynaphthalene methylene sulfonates or methylenebis(naphthalene sulfonates) of various molecular weights, obtained by polycondensation of naphthalene sulfonic acids with formaldehyde and subsequent neutralization with sodium hydroxide. The length of the polymer chains is up to 25 structural links. Polynaphthalene methylene sulfonates are linear polymers with sulfonate groups repeated at regular intervals. 
The S-3 additive is introduced into the suspension in the form of a dry substance. The chloride content is less than $0.1 \%, \mathrm{pH} 8.0$. The $\mathrm{S}-3$ additive is characterized by an electrostatic and steric mechanism of action.

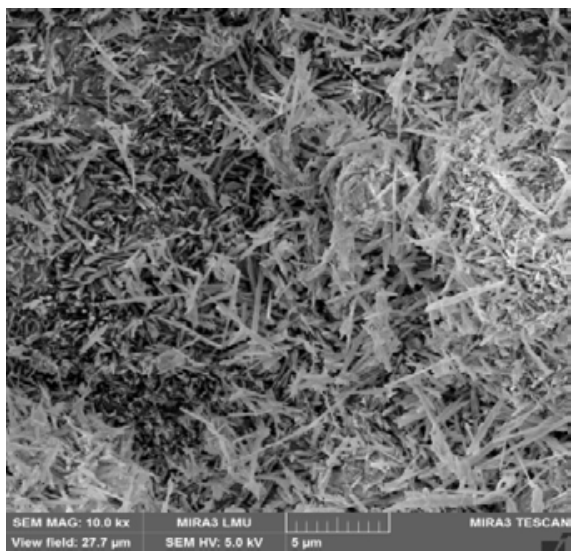

$\mathbf{a}$

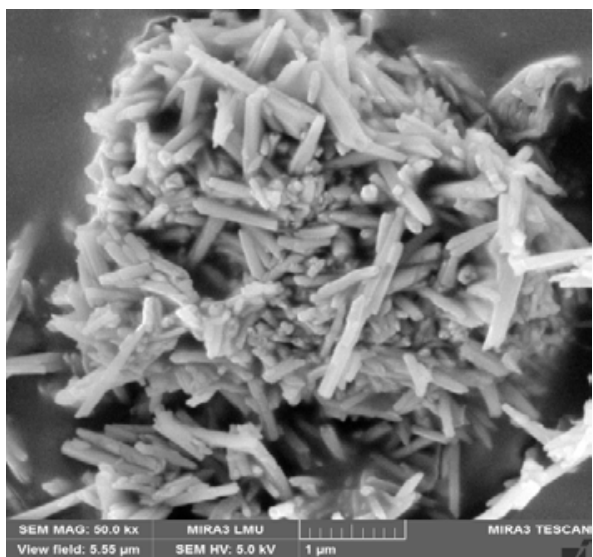

b

Fig. 1. Halloysite nanotubes: $\mathrm{a}-\mathrm{a}$ general form $\mathrm{x} 10 ; \mathrm{b}$ - an aggregate of halloysite nanotubes $\mathrm{x} 50$.

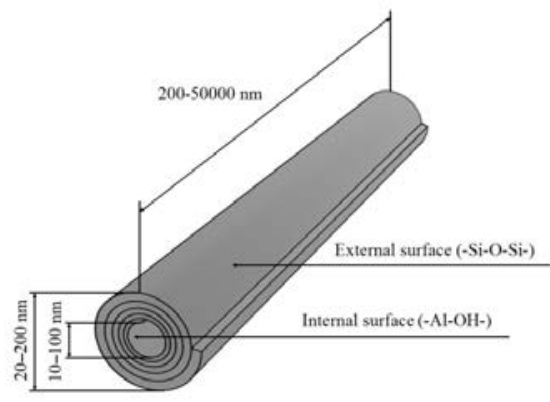

a

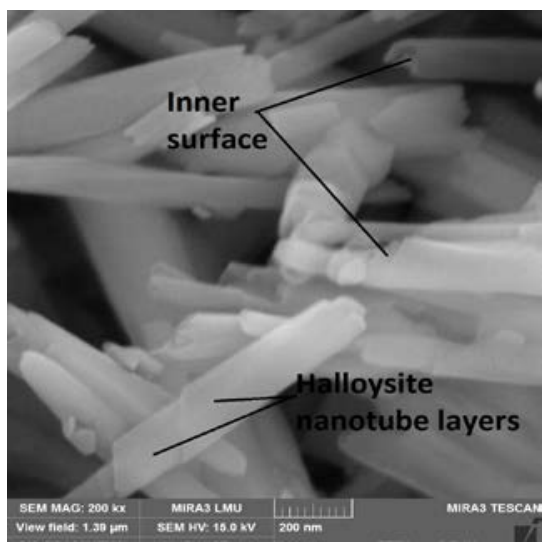

b

Fig. 2. Halloysite nanotubes: a - a schematic structure; b-SEMx200.

The MG additive is a synthetic compound based on a light brown polycarboxylate ether with a density of $1060 \mathrm{~kg} / \mathrm{m}^{3}$ (at $20^{\circ} \mathrm{C}$ ), $\mathrm{pH} \mathrm{5.8,} \mathrm{an} \mathrm{alkali} \mathrm{content} \mathrm{of} \mathrm{less} \mathrm{than} 0.6 \%$ and chlorides of less than $0.01 \%$. The $\mathrm{MG}$ additive belongs to polycarboxylates. It is characterized by a high effect of the steric and electrostatic mechanism of action.

The distilled water with a hydrogen $\mathrm{pH}$ of 6.6 served as the dispersion medium.

The suspensions were obtained using the methods below.

Variant 1. All components were added at once and treated with ultrasound for 8 minutes.

Variant 2. The stabilizer was introduced in two steps. Half of the stabilizer was mixed with water and HN. The water suspension was treated with ultrasound for 4 minutes. After having added the second half of the stabilizer, ultrasonic treatment lasted 4 minutes. 
Variant 3. The stabilizer was introduced in 3 steps. The third part of the additive was mixed with water and $\mathrm{HN}$, the ultrasonic treatment of the water suspension was carried out for 4 minutes, followed by the addition of the second third of the stabilizer. After $4 \mathrm{~min}$ ultrasonic treatment the third part of the stabilizer was added and treated with ultrasound for another 4 minutes.

The factor determining the dispersion stability and its coagulation susceptibility is the $\zeta$ potential. According to GOST R 8.887-2015, the value of the $\zeta$-potential equal to $\pm 30 \mathrm{mV}$ is a characteristic for the conditional division of low-charged and high-charged surfaces. The greater the $\zeta$-potential is, the stabler the colloidal system is [10].

To evaluate the stability to aggregation and sedimentation of the halloysite nanotubes dispersion system, the ZetaPlus analyzer was used for the $\zeta$-potential, particle size, and their specific surface area.

As shown in Table 1, the $\zeta$-potential depends on the stabilizer type and the method of its introduction into the dispersed system. The maximum values of the $\zeta$-potential of $52.9 \mathrm{mV}$ and $43.8 \mathrm{mV}$ (in magnitude) were obtained for the HN suspension stabilized with S-3 with its fractional introduction (compositions 3 and 4). This is confirmed by the electrostatic mechanism of stabilization of the halloysite nanotubes suspension by the S-3 additive. As for the MG stabilizer, the values of the $\zeta$-potential do not exceed $20.9 \mathrm{mV}$ (variant 2).

It is established that when the stabilizer is introduced into the $\mathrm{HN}$ suspension, a change in the $\zeta$-potential value is observed due to its adsorption on the surface of the halloysite nanotubes. The most intensive change in the $\zeta$-potential takes place at the initial stage. Then the $\zeta$-potential slightly changes and shortly after reaches a constant value, thus saturating the adsorption layers.

Table 1. Characteristics of the dispersion system of halloysite nanotubes with different types of stabilizers

\begin{tabular}{|c|c|c|c|c|c|}
\hline № & $\begin{array}{l}\text { Suspension } \\
\text { components }\end{array}$ & $\begin{array}{l}\zeta \text { - potential } \\
\text { (in absolute } \\
\text { value), } \mathrm{mV}\end{array}$ & $\begin{array}{c}\text { Particle } \\
\text { average size, } \\
\mu \mathrm{m}\end{array}$ & $\begin{array}{c}\text { Particle modal } \\
\text { size, } \\
\mu \mathrm{m}\end{array}$ & $\begin{array}{c}\begin{array}{c}\text { Specific } \\
\text { surface area, } \\
\mathrm{cm}^{2} / \mathrm{g}\end{array} \\
\end{array}$ \\
\hline 1 & $\begin{array}{l}\text { HN without a } \\
\text { stabilizer }\end{array}$ & 16.1 & 32.3 & 48.6 & 4993 \\
\hline 2 & $\begin{array}{c}\mathrm{HN}+\mathrm{S}-3 \\
\text { UST-time: } 8 \mathrm{~min}\end{array}$ & 28.6 & 21.2 & 30.4 & 5132 \\
\hline 3 & $\begin{array}{l}\mathrm{HN}+\mathrm{S}-3 \\
\text { UST-time: } 8 \mathrm{~min} \\
\quad(4+4)\end{array}$ & 52.9 & 9.5 & 12.9 & 11321 \\
\hline 4 & $\begin{array}{c}\mathrm{HN}+\mathrm{S}-3 \\
\text { UST-time: } 12 \mathrm{~min} \\
(4+4+4)\end{array}$ & 43.8 & 14.7 & 17.9 & 9444 \\
\hline 5 & $\begin{array}{c}\mathrm{HN}+\mathrm{MGL} \\
\text { UST-time: } 8 \mathrm{~min}\end{array}$ & 9.8 & 23.3 & 25.6 & 4980 \\
\hline 6 & $\begin{array}{l}\text { HN+MGL } \\
\text { UST-time: } 8 \text { min } \\
\quad(4+4)\end{array}$ & 20.9 & 15.1 & 18.1 & 9356 \\
\hline 7 & $\begin{array}{c}\mathrm{HN}+\mathrm{MGL} \\
\text { UST-time: } 12 \mathrm{~min} \\
(4+4+4)\end{array}$ & 15.9 & 11.7 & 15.2 & 10726 \\
\hline
\end{tabular}


The S-3 molecules with a negative charge can be adsorbed on the local positively charged areas of the inner surface of the halloysite nanotubes within the defects, chips, and exposed edges of the tubes. Some of the S-3 sulfonate groups are connected to the solid phase, while the rest are directed towards the liquid phase.

According to the findings such adsorption causes an increase in the $\zeta$-potential and improves the aggregate particle stability owing to electrostatic repulsive forces (Table 1). Furthermore, the stabilization occurs due to the spatial obstacles to aggregation resulted from the action of adsorption-solvate and structural-mechanical factors.

The fractional nature of the stabilizer introduction leads to an increase in the $\zeta$ potential values as compared to the one-step introduction of the stabilizer and ultrasound treatment for 8 minutes. In the initial stage, when halloysite nanotubes and a stabilizer are introduced into the distilled water, the latter is adsorbed on the surface of the halloysite nanotubes aggregates. Under the influence of ultrasound the HN aggregates are destroyed, new interface areas are formed, and the stabilizer molecules adsorb on them. With the onestep introduction of the S-3 stabilizer the average particle size was 21.2 microns, the modal size - 30.4 microns, the specific surface area $-5132 \mathrm{~cm}^{2} / \mathrm{g}$, and the $\zeta$-potential $-28.6 \mathrm{mV}$.

In variant 2 the introduction of the stabilizer into the dispersed system initially leads to its adsorption on halloysite nanotubes aggregates too. When they are destroyed by ultrasound, new interfaces are formed. At that, the redistribution of stabilizer molecules or its side chains on the chips and halloysite nanotubes surfaces is possible. The introduction of the second half of the stabilizer after 4 minutes of ultrasonic treatment causes its adsorption on the newly formed surfaces or the chips of the halloysite nanotubes. The average particle size decreases 3.4 times from 21.2 microns to 9.5 microns, the modal size gets 3.9 times lower from 48.6 microns to 12.9 microns, the specific surface area grows 2.3 times from 4993 to $11321 \mathrm{~cm}^{2} / \mathrm{g}$, the $\zeta$-potential increases 3.3 times from 16.1 to $52.9 \mathrm{mV}$.

As compared to variant 2, the fractional introduction of the S-3 stabilizer in variant 3 leads to lowering of the $\zeta$-potential from 52.9 to $43.8 \mathrm{mV}$, an increase in the size of the halloysite nanotubes from 9.5 to 14.7 microns, a decrease in the specific surface area from 11321 to $9444 \mathrm{~cm}^{2} / \mathrm{g}$, as the destabilization of the dispersed system confirms.

The $\zeta$-potential values, obtained for HN suspensions stabilized with S-3, are two or more times higher than the values for HN suspensions stabilized with MG. It shows that the electrostatic factor of aggregate stability for MG-stabilized suspensions is not the crucial factor. The aggregate stability is provided mainly due to the adsorption-solvation and structural-mechanical factors. In addition to the main chain, directly involved in the formation of the adsorption layer, polycarboxylates have side chains that create a spacing effect in it, making the stabilized system more stable.

The application of the MG stabilizer will result in the formation of quite compact and extensive adsorption layers leading to an increase in the apparent size of the dispersed phase particles.

Therefore, the study of the stabilization of the halloysite nanotubes dispersed system for silicate constructional materials showed the following.

1. The most preferable variant of introducing a stabilizer into the $\mathrm{HN}$ dispersed system is variant 2, when the stabilizer is added in two steps. In this case, the halloysite nanotubes have got minimal dimensions.

2. Both of the stabilizers considered (S-3 and MG) are effective for improving the aggregate stability of the dispersed system of halloysite nanotubes.

3. It is established that the $\zeta$-potential value depends not only on the stabilizer type, but also on the method of its introduction into the dispersed system. The maximum values of the $\zeta$-potential of $52.9 \mathrm{mV}$ and $43.8 \mathrm{mV}$ were obtained for the $\mathrm{HN}$ suspension stabilized with S-3 being introduced fractionally (variants 2 and 3 ). The domination of the electrostatic mechanism of stabilization of the $\mathrm{HN}$ dispersed system proves it. 
3. For the dispersed system stabilized with MG, the $\zeta$-potential values do not exceed $20.9 \mathrm{mV}$, while the particle sizes decrease and the specific surface area increases. This confirms the formation of spatial obstacles to aggregation due to the action of adsorptionsolvate and structural-mechanical factors. The electrostatic factor of aggregate stability for these dispersed systems is not crucial.

4. Taking into account the difference in charges on the inner and outer surfaces of halloysite nanotubes, it is reasonable to continue further researches in order to obtain aggregatively stable aqueous dispersed systems for a long time.

\section{References}

1. D.D. Kotelnikov, A.I. Konyukhov, Clay minerals of sedimentary rocks (1986)

2. Rock encyclopaedia (1: Aa-lava-Geosystem, 1984)

3. A.Yu. Egorov, Prospect and protection of mineral resources. 3, 19-24 (2015)

4. Y. Lvov, A. Aerov, R. Fakhrullind, Adv. Colloid Interface Sci. 207, 189-98 (2014)

5. E. Joussein, S. Petit, J. Churchman et al. Clay Minerals. 40, 383-426 (2005)

6. T.F. Bates, F.A. Hildebrand, A. Swineford. Am. Miner. 35, 463 (1950)

7. B. Singh. Clays Clay Miner. 44, 191 (1996)

8. E. Abdullayev, A. Joshi, W. Wei, Y. Zhao, Y. Lvov. ACS Nano 6, 7216 (2012)

9. G. Cavallaro, G. Lazzara, S. Milioto. J. Phys. Chem. C 116, 21932 (2012)

10. GOST 8.887-2015 (ISO 13099:2-2012, NEQ) (Standartinform, 2015) 